\title{
Emerging biology of sphingosine-1-phosphate: its role in pathogenesis and therapy
}

\author{
Richard L. Proia' and Timothy Hla² \\ ${ }^{1}$ Cenetics of Development and Disease Branch, National Institute of Diabetes and Digestive and Kidney Diseases, NIH, Bethesda, Maryland, USA. ${ }^{2}$ Center for Vascular Biology, Department of Pathology and \\ Laboratory Medicine, Weill Cornell Medical College, Cornell University, New York, New York, USA.
}

\begin{abstract}
Membrane sphingolipids are metabolized to sphingosine-1-phosphate (S1P), a bioactive lipid mediator that regulates many processes in vertebrate development, physiology, and pathology. Once exported out of cells by cell-specific transporters, chaperone-bound S1P is spatially compartmentalized in the circulatory system. Extracellular S1P interacts with five GPCRs that are widely expressed and transduce intracellular signals to regulate cellular behavior, such as migration, adhesion, survival, and proliferation. While many organ systems are affected, S1P signaling is essential for vascular development, neurogenesis, and lymphocyte trafficking. Recently, a pharmacological S1P receptor antagonist has won approval to control autoimmune neuroinflammation in multiple sclerosis. The availability of pharmacological tools as well as mouse genetic models has revealed several physiological actions of S1P and begun to shed light on its pathological roles. The unique mode of signaling of this lysophospholipid mediator is providing novel opportunities for therapeutic intervention, with possibilities to target not only GPCRs but also transporters, metabolic enzymes, and chaperones.
\end{abstract}

\section{Introduction}

Bioactive lipids, derived from metabolism of plasma membrane lipids, are important mediators of cellular communication in vertebrates. The appearance of bioactive lipid receptors in the vertebrate genomes (1) was coincident with the increased complexity of circulatory, immune, and nervous systems in evolution, suggesting that vertebrates began to use extracellular signaling of lipid mediators for the regulation of sophisticated organ systems. This Review will focus on the lysosphingolipid sphingosine-1phosphate (S1P) and how the basic understanding of its metabolism, transport, and signaling functions has revealed its role in the pathogenesis of various diseases and allowed rational therapeutic strategies to advance.

\section{S1P metabolism}

Sphingosine, the precursor substrate for the synthesis of S1P, is derived by the hydrolysis of ceramide during the sequential degradation of plasma membrane glycosphingolipids and sphingomyelin (refs. 2, 3, and Figure 1). Even though this occurs in various cell compartments, the bulk of sphingosine is generated by degradation in lysosomes. Indeed, the prominence of this lysosomal catabolism pathway is illustrated by the severity of the sphingolipidoses, a family of genetic disorders in which sphingolipid metabolites accumulate (4). The catabolically generated sphingosine is phosphorylated by either of two sphingosine kinases, SPHK1 and SPHK2, to produce S1P. SPHK1 is largely cytoplasmic and can acutely associate with the plasma membranes (5), phagosomes (6), and endosomal vesicles (7), whereas SPHK2 is present cytoplasmically but is predominately in the nucleus (8).

Conflict of interest: The authors have declared that no conflict of interest exists. Reference information: J Clin Invest. 2015;125(4):1379-1387. doi:10.1172/JCI76369.
While not strictly required for cellular viability (9), the formation of S1P is essential for organismal development (10). The viability of the single Sphk KO mice $(10,11)$ indicates that the isozymes can partially compensate for each other during development but have nonoverlapping functions.

Once formed intracellularly, S1P takes one of three pathways (Figure 1). In one, the sphingosine moiety of S1P is recycled through ceramide synthesis after dephosphorylation by S1P-specific ER phosphatases, SGPP1 and SGPP2 $(12,13)$. In some mammalian cells, this pathway can account for greater than half of complex sphingolipid synthesis (14).

In a second pathway, S1P is irreversibly degraded by S1P lyase, another ER-resident enzyme, into phosphoethanolamine and hexadecenal (15). This reaction facilitates transfer of substrate from the sphingolipid to the glycerolipid pathway via the conversion of hexadecenal by fatty aldehyde dehydrogenase to hexadecanoate, a precursor of palmitoyl-CoA (16), and by the utilization of phosphoethanolamine for phosphatidylethanolamine synthesis $(17,18)$.

In the third pathway, intracellular S1P is released to the extracellular environment, a process that is highly efficient in rbc (19-21), platelets (22), and endothelial cells (19-21). A specific S1P transporter, SPNS2, is used in endothelial cells for S1P secretion (23). The precise secretion mechanism in rbc has not been established, but it does not involve SPNS2 (23). In platelets, S1P is exported after activation by thrombotic agonists (24). The biochemical pathways and cellular localization of S1P metabolism and release are illustrated in Figure 1.

\section{Compartmentalized enrichment of chaperone- bound S1P in circulation}

S1P concentrations are elevated in plasma $(\sim 1 \mu \mathrm{M})$ and lymph $(\sim 100 \mathrm{nM})$ relative to the interstitial fluid of tissues. This S1P gradient is essential for many of the physiologic functions pro- 


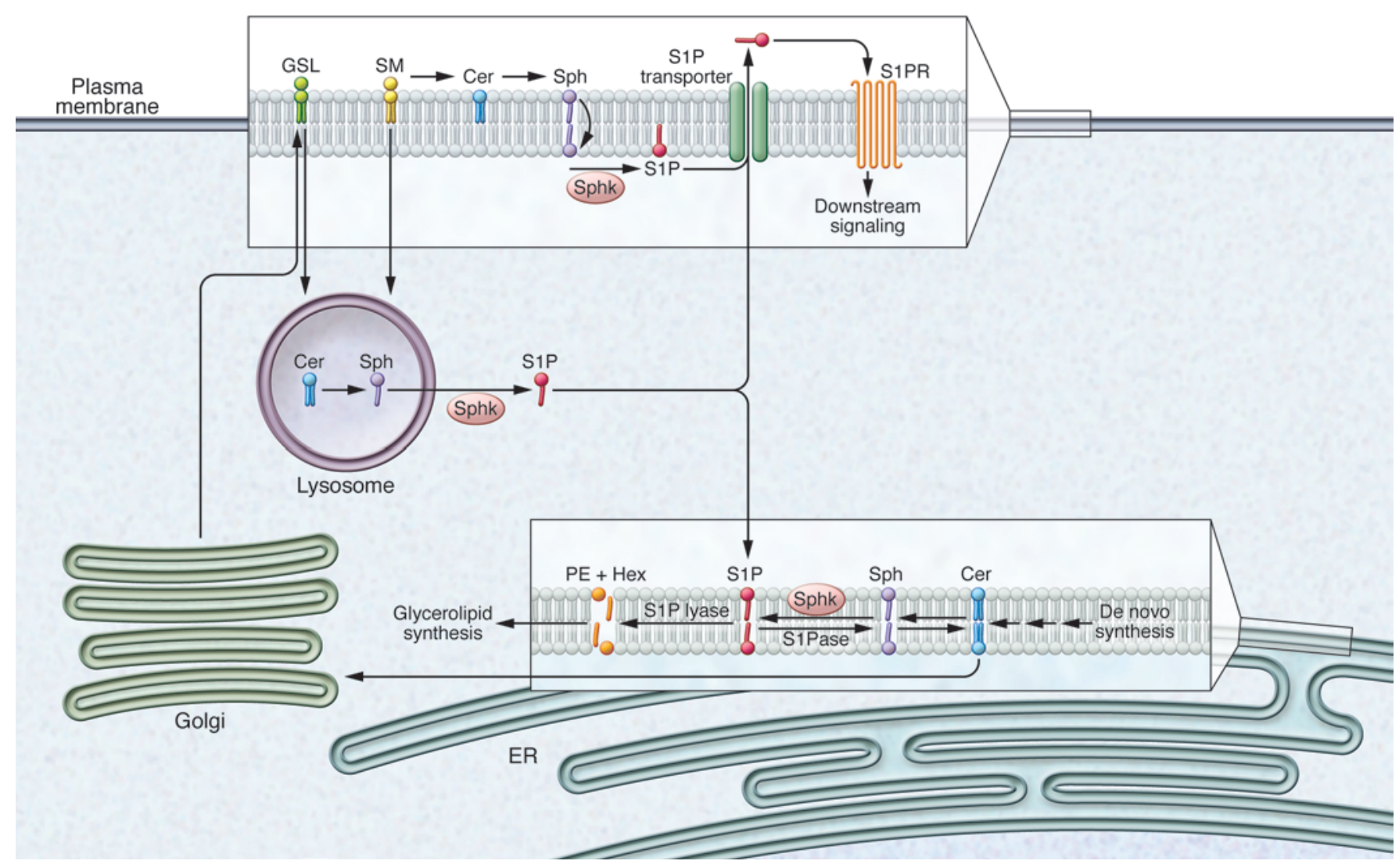

Figure 1. S1P synthesis, metabolism, and export. Sphingomyelin (SM) and glycosphingolipids (GSL) are localized to plasma membrane microdomains. Degradation of plasma membrane sphingolipids into ceramide (Cer) and then ultimately to sphingosine (Sph) occurs in lysosomes and at the plasma membrane. Phosphorylation mediated by sphingosine kinases leads to the formation of S1P, which follows one of three pathways: (a) S1P is exported by transporters, such as SPNS2, where it can interact with its GPCRs $\left(\mathrm{S1P}_{1}-\mathrm{S1P}_{5}\right.$ ) on the cell surface; (b) S1P is degraded by S1P lyase into hexadecenal (Hex) and phosphoethanolamine (PE), which are shunted into the glycerolipid biosynthetic pathway; or (c) S1P is dephosphorylated by S1P phosphatase for recycling of sphingosine into ceramide synthesis. S1PR, S1P receptor; S1Pase, S1P phosphatase.

vided by extracellular S1P (25). High levels of S1P in circulation are a result of rbc and endothelial cells, which are metabolically geared toward S1P secretion (25). Indeed, rbc produce almost all embryonic S1P (26) and approximately $75 \%$ of adult plasma S1P in mice $(21,26)$. The vascular endothelium is another key contributor (19), whereas platelets are not critical for plasma S1P concentrations in postnatal homeostatic conditions $(19,27)$ and may only release S1P during platelet activation and clotting. The lymphatic endothelium is the major source of lymph S1P (ref. 28 and Figure 2).

S1P lyase expression is essential for maintenance of low levels of S1P within tissues; in its absence, levels of S1P in tissue are highly elevated $(29,30)$. The lipid phosphatase LPP3, while not determinative of bulk tissue S1P levels, appears to control local levels around the sites of lymphocyte egress in the thymus (31).

Within the plasma, most S1P is bound to protein carriers, such as HDL $(\sim 60 \%)$ and albumin $(\sim 30 \%)$, with lesser amounts bound to VLDL and LDL (32). S1P is bound to HDL via the apolipoprotein ApoM, which acts as an S1P chaperone that controls the levels of S1P in blood (33). Chaperones enable aqueous solubility of S1P and allow it to be transported as a paracrine and endocrine mediator. In addition, chaperones such as ApoM may also protect S1P from degradation and facilitate presentation to receptors.

\section{S1P receptors}

Extracellular S1P interacts with a family of five high-affinity GPCRs, termed S1P $-{\mathrm{S} 1 \mathrm{P}_{5}}_{5}(34)$. The S1P receptors, especially $\mathrm{S}_{1} \mathrm{P}_{1}-$ $\mathrm{S}_{1} \mathrm{P}_{3}$, are broadly expressed in tissues (35). $\mathrm{S}_{1} \mathrm{P}_{1}$ is notable as one of the most highly expressed GPCRs in the entire super family (35-37). The very high expression of $\mathrm{S}_{1} \mathrm{P}_{1}$ on endothelial cells can partially explain its wide tissue distribution; however, many other cell types also express this GPCR.

Each of the receptors, when activated, provokes distinctive signaling pathways and cellular responses that are, in some cases, antagonistic. Other recent reviews discuss this subject in depth $(34,38)$.

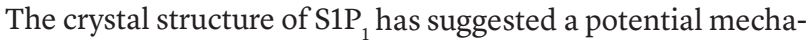
nism for ligand access to the GPCR that involves insertion of S1P into the outer leaflet of the plasma membrane followed by lateral diffusion to enter the binding pocket (39). This suggests that phosphatases close to the S1P receptors could regulate ligand access. In addition, S1P chaperones may impart or enhance specific biological signals. For example, ApoM-bound S1P is much more effective at activating endothelial S1P receptors in the control of lung vascular barrier function than albumin-bound S1P (33). How various chaperones present S1P to their receptors and how S1P is released at the cell surface from chaperones is not known. 

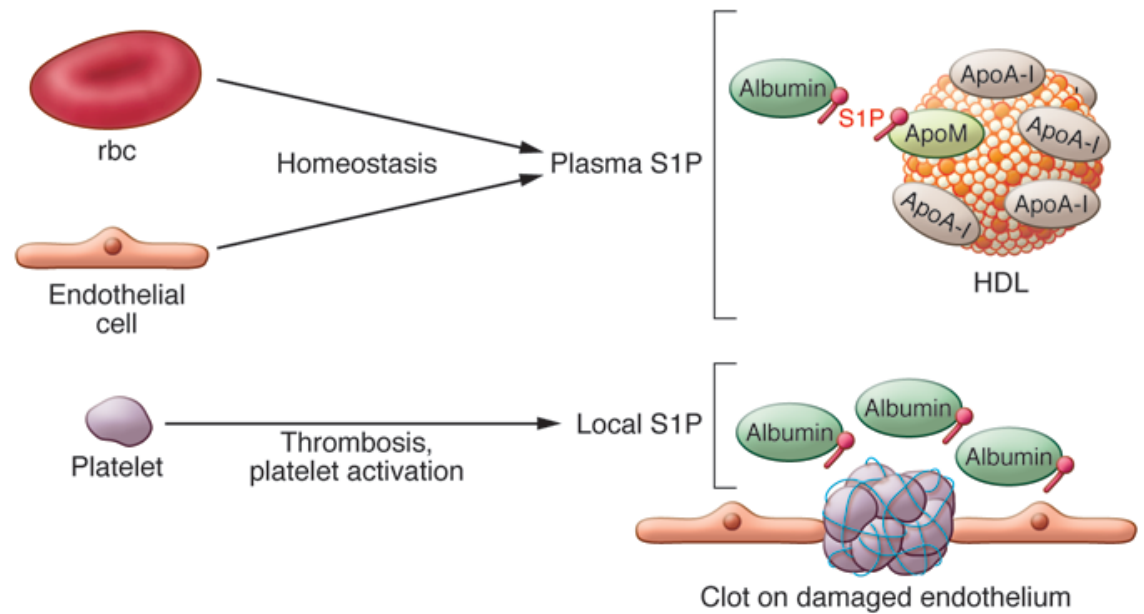

Figure 2. Cellular sources of plasma S1P. Endothelial cells and rbc release S1P, which is picked up and chaperoned by ApoM on the HDL particles and albumin. Chaperone-bound S1P interacts with S1P on the endothelial cells to promote vascular barrier function. When endothelial cells are damaged, platelet activation and aggregation release S1P, which leads to local release of S1P that aids in the repair of vascular injury.

\section{S1P as a regulator of vascular development and function}

$\mathrm{S}_{1} \mathrm{P}_{1}, \mathrm{~S}_{2} \mathrm{P}_{2}$, and $\mathrm{S}_{1} \mathrm{P}_{3}$ are expressed in endothelial cells, in which they regulate development and function of the vasculature (40, 41). $\mathrm{S}_{1} \mathrm{P}_{1}$ expression in endothelial cells is essential for the development of the embryonic cardiovascular system, whereas $\mathrm{S}_{1} \mathrm{P}_{2}$ and $\mathrm{S}_{1} \mathrm{P}_{3}$ appear to play accessory or partially redundant roles. Likewise, S1P production by rbc is required for embryonic vascular development, highlighting the unique communication between rbc and endothelial cells (ref. 26 and Figure 3).

S1P signaling has a fundamental function in the maintenance of vascular integrity, even in the postnatal period. Mice that are engineered to lack plasma S1P show vascular leakage, which is reversed by an $\mathrm{S}_{1} \mathrm{P}_{1}$ agonist (27). The results are consistent with the idea that S1P within the vascular compartment acts on endothelial S1P ${ }_{1}$ to minimize vascular leak during homeostatic and acute conditions (Figure 3). S1P signaling reporter mice demonstrate receptor activation in endothelium under basal conditions, with enhanced S1P activation, dependent on plasma S1P, when systemic inflammation is induced (42).

$\mathrm{S} \mathrm{P}_{2}$ and $\mathrm{S}_{1} \mathrm{P}_{3}$ are also expressed in vascular smooth muscle cells of resistance vessels and regulate vascular tone, especially during endothelial damage when plasma S1P accesses the medial smooth muscle cells (43-47). Alternatively, during injury or activation of vascular smooth muscle, enhanced activation/expression of sphingosine kinases could induce local S1P production within the vessel wall to influence vascular tone. In specialized vasculature, such as the inner modiolar artery that supplies blood to the inner ear structures, $\mathrm{S}_{2} \mathrm{P}_{2}$ appears to be used in the control of vascular tone. Indeed, mice that lack ${\mathrm{S} 1 \mathrm{P}_{2}}_{2}$ show stria vascularis abnormalities, inner ear defects, and deafness (48-50).

\section{S1P as a regulator of hematopoietic cell trafficking}

S1P receptor-mediated sensing of elevated S1P levels in the blood and lymph serves as a general mechanism to trigger the egress of hematopoietic cells from tissues into the circulation (Figure 3). S1P $P_{1}$ expression on $\mathrm{T}$ and $\mathrm{B}$ cells stimulates their exit from lymphoid tissues, with low S1P levels, into blood or lymph, with high S1P levels (51). Hematopoietic stem cell mobilization from the bone marrow into the blood and recirculation from tissues to bone marrow are also facilitated by S1P function $(35,52,53)$. In a similar manner, $\mathrm{S}_{5}$ regulates the egress of $\mathrm{NK}$ cells from bone marrow to blood (54). Interestingly, $\mathrm{S}_{1} \mathrm{P}_{1}$ on megakaryocytes triggers the elongation of transendothelial proplatelet extensions into bone marrow sinusoids and the subsequent shedding of platelets into the circulation (55). Although, S1P signaling affects monocyte and neutrophil levels in blood, the precise control points have not yet been elucidated $(30,56)$.

$\mathrm{T}$ cell trafficking through the lymph node serves as a paradigm that illustrates some basic features of the S1P-regulated egress process (51). While in blood, in which it is exposed to high S1P concentrations, $\mathrm{S1P}_{1}$ is rapidly downregulated through GPCR kinase-2mediated (GRK2-mediated) phosphorylation of a serine-rich region on its C-terminal tail $(57,58)$. After entering lymph nodes through high endothelial venules (HEVs), $\mathrm{S}_{1} \mathrm{P}_{1}$ is reexpressed while in the low S1P environment of the lymph node parenchyma. The S1P ${ }_{1}$-expressing T cell "probes" near the cortical sinus endothelium for the presence of S1P. If S1P/S1P signaling strength is sufficient to overcome parenchymal retention signals, the $\mathrm{T}$ cell traverses the endothelium to enter the efferent lymphatics (Figure 3).

S1P receptor signaling also has roles in the positioning and migration of cells within tissues. Some key examples include S1P's role in $B$ cell shuttling between the marginal zone and follicles (59) and S1P ${ }_{2}$ 's role in confining B cells in the center of follicles as well as regulating their proliferation (60). In this last example, $\mathrm{S}_{1} \mathrm{P}_{2}$ serves as a tumor suppressor; when S1pr2 is deleted, mice eventually develop diffuse large B cell lymphoma (DLBCL) (61), and S1PR2 mutations occur in some cases of human DLBCL (62-64). The $\mathrm{S1P}_{2}$ mutations in human DLBCL block the inhibitory effects on phospho-AKT and migration pathways mediated by $\mathrm{S}_{2} \mathrm{P}_{2}(64)$.

Fingolimod (FTY720), a sphingosine analog, has been an important chemical probe for understanding S1P-directed immune cell trafficking through its "functional antagonism" of $\mathrm{S}_{1} \mathrm{P}_{1}(65)$. FTY720 is phosphorylated primarily by SPHK2 in vivo to produce FTY720-phosphate, a potent, acute agonist for $\mathrm{S}_{1} \mathrm{P}_{1}, \mathrm{~S}_{3} \mathrm{P}_{3}$, ${\mathrm{S} 1 \mathrm{P}_{4}}_{4}$, and $\mathrm{S1P}_{5}$. However, FTY720-phosphate-induced downmodulation of $\mathrm{S}_{1} \mathrm{P}_{1}$ from the lymphocyte cell surface blocks receptor signaling, inhibiting the egress of lymphocytes from lymphoid tissues into the circulation. Primarily due to its effect on trafficking of 
A

\section{Embryonic development}

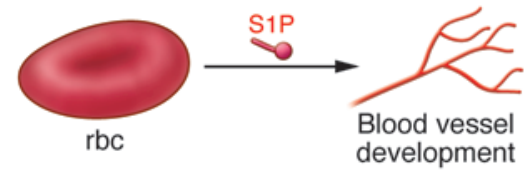

Adult vascular homeostasis

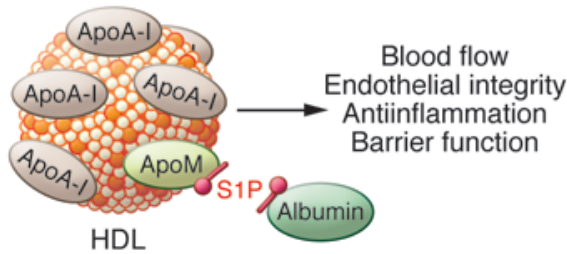

B

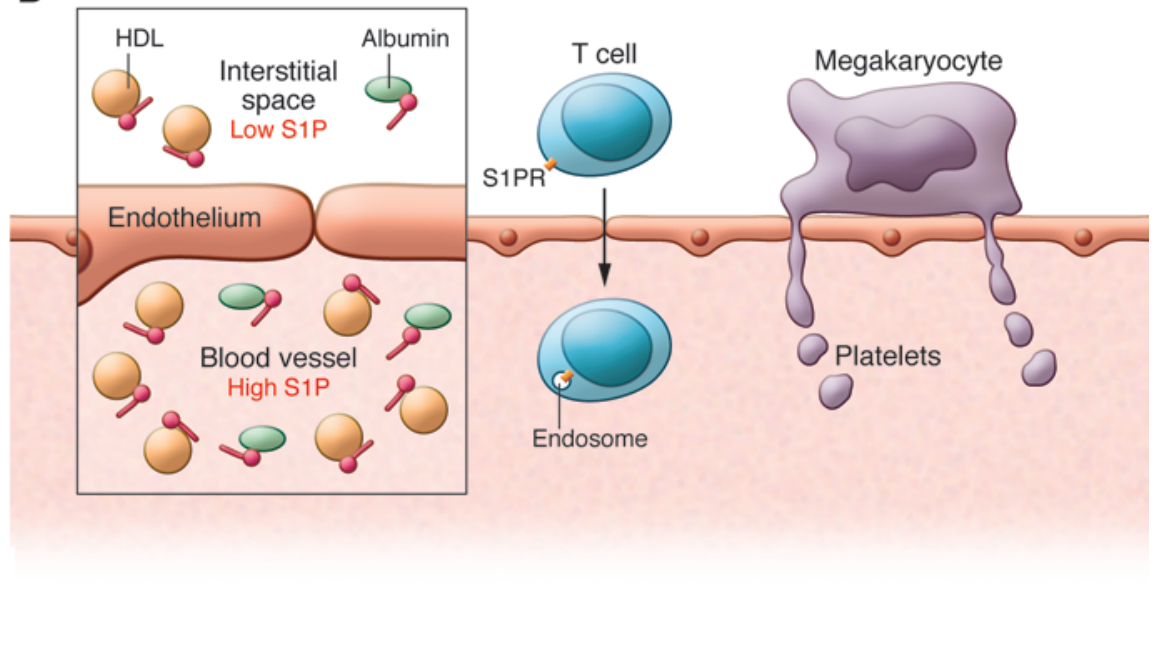

Figure 3. Functions of S1P in vascular development and hematopoietic cell trafficking. (A) During embryogenesis, rbc-derived S1P plays an essential role in the stabilization of the developing vascular system. In the postnatal period, both rbc and the endothelium release S1P into circulation to maintain vascular homeostasis. Plasma S1P is associated with ApoM+ $\mathrm{HDL}$ and albumin. S1P signaling regulates blood flow, endothelial integrity, barrier function, and antiinflammatory functions. (B) There is a steep S1P gradient between vascular and extravascular compartments. This is essential for trafficking of T cells from thymus and secondary lymphoid organs into blood and lymph, respectively. In the bone marrow sinusoids, S1P signaling is important for the release of megakaryocyte proplatelet extensions into the circulation.

autoreactive adaptive immune cells, FTY720 is a potent inhibitor of autoimmune inflammation (see below).

Regulators of S1P levels also affect lymphocyte trafficking. Mice that lack the S1P transporter SPNS2, which functions in endothelial cells to secrete S1P into the circulation (especially into the lymph fluid), exhibit profound lymphopenia due to a decreased egress of lymphocytes into the circulation $(23,66-68)$. Mice with S1P lyase deleted or pharmacologically inhibited are also lymphopenic due to impaired lymphocyte trafficking $(29,30$, 69). However, in this case, the cause is elevated tissue levels of S1P disrupting the concentration differential between lymphoid tissues and the circulation. A partial deficiency of S1P lyase protects mice from experimental autoimmune encephalomyelitis (EAE) (70). Potentially, inhibitors of both the SPNS2 transporter and S1P lyase could be used to modulate immune pathologies.

\section{S1P signaling controls nervous system development}

$\mathrm{S}_{1} \mathrm{P}_{1}$ signaling is active in the developing nervous system (42), and both S1P synthesis and S1P expression are required for embryonic neurogenesis (10). In the adult nervous system, multiple S1P receptors are expressed on both neurons and glia (71) and may regulate neurotransmission (72-74). A key function of $\mathrm{S1P}_{1}$ and $\mathrm{S1P}_{3}$ occurs during inflammatory disease processes through the promotion of astrogliosis $(75,76)$. S1P receptor signaling induces nociceptive responses, possibly as a result of local increases of S1P, which are induced by injury or inflammation and sensed by $\mathrm{S}_{3} \mathrm{P}_{3}$ $(35,77-79)$. Overall, the transport and biological functions of S1P in the nervous system are not well understood.

\section{Mapping in vivo S1P signaling sites}

A reporter mouse model that transcriptionally records $\mathrm{S}_{1} \mathrm{P}_{1}$ activation has enabled the mapping of cellular sites of activated S1P ${ }_{1}$ in vivo (42). A synthetic $\mathrm{S}_{1} \mathrm{P}_{1}$ signaling pathway was genetically engineered in these mice so that a transcription factor is released from activated $\mathrm{S}_{1} \mathrm{P}_{1}$ to turn on a GFP reporter gene. In embryos from these reporter mice, $\mathrm{S1P}_{1}$ activation was apparent in the developing cardiovascular nervous system and CNS. In adult signaling reporter mice, the major cellular sites displaying activated $\mathrm{S}_{1} \mathrm{P}_{1}$ were endothelial cells, with the vascular and lymphatic endothelial cells of lymphoid tissues showing the most prominent levels of S1P activation. Intense S1P activation was found on HEVs and is likely indicative of a process through which this specialized endothelium retains vascular integrity in the face of extensive lymphocyte transmigration. In lymph nodes with high leukocyte traffic, HEV S1P ${ }_{1}$ activation occurs by local release of S1P from extravasated platelets in the perivenular spaces, promoting vascular integrity via adherens junction assembly (80).

Parenchymal cells of tissues, such as hepatocytes in liver, did not demonstrate significant $\mathrm{S}_{1} \mathrm{P}_{1}$ activation under basal conditions, suggesting, that under normal circumstances, $\mathrm{S}_{1} \mathrm{P}_{1}$ receptors are held in reserve for signaling. However, when systemic inflammation was induced by administration of bacterial LPS, S1P $_{1}$ signaling was substantially increased in both the endothelium and hepatocytes of the liver. This increase in $\mathrm{S1P}_{1}$ activation by LPS was shown to be dependent on hematopoietically derived S1P.

The mechanism whereby S1P $\mathrm{P}_{1}$ signaling in endothelial cells is increased during inflammatory conditions and vascular leakage is not completely understood but may involve the sensing of abluminally deposited S1P from the circulation (27).

Inflammation-induced $\mathrm{S}_{1} \mathrm{P}_{1}$ signaling in liver hepatocytes and, presumably, in other tissue parenchymal cells is also mediated by hematopoietically derived S1P, indicating that this S1P signaling pool likely comes from the plasma compartment entering the tissues through vascular leakage. The functional consequences 
of receptor signaling by extravasated S1P on parenchymal and immune cells in tissues under acute and chronic inflammatory conditions is not well understood but could potentially lead to reparative processes. Whether dysregulated S1P signaling in tissues leads to fibrotic responses is not yet known; however, $\mathrm{S}_{2} \mathrm{P}_{2}$ and $\mathrm{S}_{1} \mathrm{P}_{3}$ have been associated with tissue fibrotic processes in organs, such as the liver, lung, and kidney (81-83).

\section{S1P in disease}

Multiple sclerosis. Multiple sclerosis (MS) is a chronic autoimmune disease characterized by blood-brain barrier break down, immune cell infiltration of the CNS, demyelination, astrogliosis, and neurodegeneration (84). S1P signaling activity is relevant for disease pathogenesis, as demonstrated by the efficacy of FTY720 for the treatment of relapsing-remitting MS (65). S1P action likely occurs at multiple cellular sites within the immune, vascular, and nervous systems (65).

$\mathrm{S}_{1} \mathrm{P}_{1}$-mediated egress of pathogenic lymphocytes from lymph nodes is a key control point in MS. FTY720 is believed to modify MS disease progress by the lymph node retention of autoreactive $\mathrm{T}$ cells, which damage the nervous system. Immune cell S1P $P_{1}$ signaling in the CNS is also involved in pathogenesis. Brain lesions from patients with MS exhibited phosphorylation of $\mathrm{S}_{1} \mathrm{P}_{1}$ serine 351 , a modification critical for receptor internalization (85). Mice with a mutation that precludes phosphorylation of this residue developed more severe Th17-mediated autoimmune neuroinflammation, revealing another potential S1P signaling process that may alter disease course in MS.

S1P receptors are widely expressed on cells of the CNS, including neurons, astrocytes, microglia, and oligodendrocytes, all of which have potential roles in the pathogenesis of MS. A direct role for $\mathrm{S}_{1} \mathrm{P}_{1}$ on astrocytes has been demonstrated in the disease progression of EAE. Deletion of $S 1 p r 1$ in astrocytes reduced the clinical severity of EAE and abrogated the diseasesuppressing effects of FTY720 (75).

MS has a much higher incidence in females than in males. $\mathrm{S}_{2} \mathrm{P}_{2}$ has been determined to account for differences in the enhanced disease susceptibility of female SJL EAE mice by virtue of its sexually dimorphic expression pattern in brain regions relevant to disease (86). In female SJL EAE mice and in female patients with MS, $\mathrm{S} \mathrm{P}_{2}$ was more highly expressed than in their male counterparts. The ability of $\mathrm{S} \mathrm{P}_{2}$ to destabilize adherens junctions (87), promote inflammation, and modulate the infiltration of leukocytes (88) may enhance the severity of the CNS autoimmunity.

Sjögren-Larsson syndrome. Sjögren-Larsson syndrome is a rare autosomal recessive neurocutaneous disorder with onset in infancy. The disease is characterized by ichthyosis, intellectual deficits, and delays in reaching motor milestones. The disease is caused by mutations in the $A L D H 3 A 2$ gene, which encodes a fatty aldehyde dehydrogenase involved in the degradation pathway of S1P (16). After cleavage of S1P by S1P lyase to produce hexadecenal, the fatty aldehyde is subsequently converted to hexadecenoic acid by ALDH3A2 and, ultimately, to palmitoyl-CoA via hexadecanoyl-CoA. The absence of ALDH3A2 results in the accumulation of toxic fatty aldehydes.

Sphingolipids are highly abundant in the skin and nervous system, which are major sites of pathogenesis in the disease. In skin, sphingolipid synthesis is essential for its barrier function (89) and, in nervous tissues, glycosphingolipid synthesis is prominent (90). It is likely that an important fraction of toxic fatty aldehydes in the patients may be derived during S1P metabolism, possibly explaining the pathogenic targeting of these tissues.

Influenza. Severe influenza infection is associated with significant morbidity and mortality, in part because of a "cytokine storm," an excessive, dysregulated immune response (91). S1P agonists substantially blunted the cytokine storm and protected against the pathogenic pulmonary effects of a human H1N1 influenza infection in mice (91) and in ferrets, a model that more closely resembles the clinical course in humans (92). A central mechanism of the protection involves $\mathrm{S}_{1} \mathrm{P}_{1}$ signaling on pulmonary endothelial cells, where the receptor is very highly expressed, to negatively regulate the excessive cytokine/chemokine response and pulmonary immune cell recruitment that occurs during influenza infection. $\mathrm{S}_{1} \mathrm{P}_{1}$ activation blunted the inflammatory signaling cascade downstream of several innate sensing pathways, including those derived from myeloid differentiation primary response gene 88 (MYD88) and IFN- $\beta$ promoter stimulator-1 signaling (MAVS, also known as IPS1). The results suggest that short-term use of $\mathrm{S}_{1} \mathrm{P}_{1}$ agonists may be therapeutic in suppressing the pathogenic cytokine storm in influenza and possibly in other acute respiratory diseases (93). However, the ability of these agents to induce lymphopenia and potentially suppress adaptive immune responses could outweigh the beneficial antiinflammatory effects in the vasculature. Agents that selectively influence $\mathrm{S}_{1} \mathrm{P}_{1}$ signaling in the endothelium without blunting adaptive immunity would be optimal.

Acute lung injury. Acute lung injury can be caused by bacterial or viral pneumonia, sepsis, ischemia/reperfusion injury, or trauma (94). A central underlying component driving pathogenesis involves endothelial barrier disruption and increases in vascular permeability. S1P infusion significantly restricted vascular leakage in LPS-induced lung injury in murine and canine models (95). Acute dosing of FTY720 also significantly reduced vascular leakage and associated inflammatory cell infiltrates in the LPSlung injury model (96).

In contrast to these findings, prolonged treatment with FTY720 or with the $\mathrm{S}_{1} \mathrm{P}_{1}$-selective agonist, AUY954, in the bleomycin mouse model of lung injury dramatically worsened vascular leakage and exacerbated fibrotic responses (82). These results are consistent with others that have shown that exposure to $\mathrm{S}_{1} \mathrm{P}_{1}$ agonists causes downmodulation of $\mathrm{S}_{1} \mathrm{P}_{1}$, disruption in $\mathrm{S} 1 \mathrm{P} / \mathrm{S}_{1} \mathrm{P}_{1}$ signaling, and vascular leakage (97). Agents that appropriately enhance $\mathrm{S}_{1} \mathrm{P}_{1}$ signaling in the vascular endothelium may provide protection from fibrotic diseases.

Sickle cell disease. In sickle cell disease, the sickling of rbc leads to severe manifestations that include hemolysis, anemia, hypoxia, inflammation, and tissue injury. In individuals and mice with sickle cell disease, S1P levels are substantially elevated in blood (98). These results suggest that hypoxia-induced changes induce rbc damage, hemolysis, and enhanced S1P levels in plasma. This may contribute to tissue injury directly as a result of the signaling activity of S1P within the context of endothelial damage and systemic inflammation present within the disorder (99). However, whether S1P released from sickled $\mathrm{rbc}$ is protective or injurious is not known. 
It was hypothesized that SPHK1 inhibition may represent a possible therapeutic strategy during sickle cell disease pathogenesis (98).

Cancer. S1P signaling regulates several of the "hallmarks of cancer," including induction of angiogenesis (100), resistance to cell death, sustained proliferative signaling, tumor-promoting inflammation $(101,102)$, and activation of invasion and metastasis (100-105). Furthermore, S1P metabolism was disturbed in several types of tumors, providing further support for a linkage between pleiotropic actions of S1P and oncogenesis (105).

The major approaches aimed at targeting S1P actions in cancer have focused on removal of extracellular S1P, the application of receptor-active compounds, and the use of sphingosine kinase inhibitors (105).

To remove extracellular S1P, a novel therapeutic reagent was formulated that makes use of a monoclonal antibody to physically sequester extracellular S1P. In animal models, the S1P-specific monoclonal antibody (Sphingomab) reduced tumor growth and demonstrated antiangiogenic effects (106).

The use of receptor-active compounds in cancer may be problematic due to the wide distribution of receptors and their pleiotropic activities, particularly immune suppression. Nonetheless, in a model of colitis-associated cancer, FTY720 was able to dampen the inflammatory S1P1/STAT3 amplification cascade by receptor downmodulation to suppress carcinoma progression (102). However, it is not known whether clinically relevant doses of FTY720 regulate disorders of the gut-associated immune system.

The use of sphingosine kinase inhibitors, with the goal of inhibiting tumor cell S1P synthesis to decrease viability and growth, has yielded contradictory results. Earlier studies with genetic models of sphingosine kinase overexpression (107) and lower potency sphingosine kinase inhibitors have suggested a potentially important role for SPHKs in tumor cell proliferation and survival (105). However, results with novel, highly potent, and selective inhibitors that reduce S1P to undetectable levels in tumor cells did not affect their growth in vitro or in vivo, suggesting that tumor sphingosine kinases may not be efficacious therapeutic targets for cancer (108-110).

Inflammatory bowel disease. Inflammatory bowel disease, which encompasses Crohn's disease and ulcerative colitis, is characterized by chronic, destructive inflammation in the gastrointestinal tract. A potential involvement of S1P is suggested by observations showing that deletion of Sphk1, which lowers S1P levels, reduced the severity of colitis (111), while deletion of Sphk2 (102), which elevates S1P levels through compensatory mechanisms, increased severity.

FTY720 as well as $\mathrm{S1P}_{1}$ subtype-specific inhibitors ameliorate the severity of symptoms in murine models of colitis, in which the disease is mediated by aberrant $\mathrm{T}$ cell responses. The mechanisms underlying the effectiveness of the treatments were shown to involve both $\mathrm{CD} 4{ }^{+} \mathrm{CD} 25^{+}$Tregs and Th2-type functions $(112,113)$.

In addition to the direct regulation of $\mathrm{T}$ cell function, S1P signaling may have an important role in the vasculature during colitis. Patients with ulcerative colitis display an increase in $\mathrm{S}_{1} \mathrm{P}_{1}$ levels in inflamed mucosa as well as elevated SPHK1 expression (114). Deletion of S1pr1 in the endothelium of mice caused colonic vascular leakage and enhanced bleeding in a mouse model of colitis, pointing to an important role for $\mathrm{S}_{1} \mathrm{P}_{1}$ signaling in maintaining vascular integrity during colitis. Importantly, therapeutic dosing of FTY720 and $\mathrm{S}_{1} \mathrm{P}_{1}$-specific compound AUY954 did not increase bleeding in the dextran sodium sulfate tissue injury model of colitis, indicating that ligand-induced receptor degradation was not functionally significant. These results imply that therapeutic modifications of $\mathrm{S}_{1} \mathrm{P}_{1}$ signaling may encompass a means to blunt the severity of the disease, since immune S1P receptors show enhanced sensitivity to pharmacological agents, whereas endothelial S1P receptors exhibit large receptor reserve.

Vascular and cardiac diseases. The role of S1P in vascular and cardiac diseases is beginning to be appreciated but is not well understood. Several S1P receptor modulators were shown to reduce inflammatory responses and atherosclerosis in mouse models $(115,116) . \mathrm{S}^{2} \mathrm{P}_{2}$ is expressed in atherosclerotic plaques and contributes to macrophage content and inflammatory responses. In models of atherosclerosis, S1pr2 knockout mice exhibited reduced pathological lesions and macrophage content, apparently because of altered S1P signaling in the myeloid

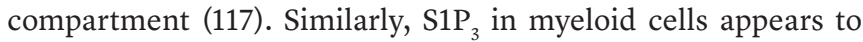
regulate myeloid cell influx to inflammatory vascular lesions (118). Even though $\mathrm{S}_{2} \mathrm{P}_{2}$ regulates endothelial cell inflammatory responses (87), its functional significance in atherosclerosis has not been addressed.

$\mathrm{S} 1 \mathrm{P}$ is produced in the heart during brief periods of ischemia and protects the myocardium $(119,120)$. It also activates the $G$ protein-coupled inwardly rectifying potassium (GIRK) channels at the sinoatrial node and induces bradycardia (121). This process is a well-known initial adverse event of $\mathrm{S}_{1} \mathrm{P}_{1}$-activating drugs but subsides after an initial dose (65). Whether S1P signaling participates in cardiac arrhythmias is not known. Furthermore, although S1P receptors are involved in cardiac hypertrophy (122), the importance of this pathway in heart failure has not been examined.

\section{Concluding remarks and future outlook}

In addition to the metabolic enzymes and receptors, newly characterized regulators of S1P signaling, such as S1P chaperones (33) and transporters (23), have illuminated new insights into the unique biology of this lipid signaling system. Such mechanistic insights have also revealed additional control points for potential therapeutic intervention. Even though receptor modulators have entered the mainstream of current pharmacopeia, the complexity and ubiquity of the signaling system likely means that targeting various steps in the pathway may allow better control of the dysregulated S1P signaling that occurs in multiple diseases.

\section{Acknowledgments}

R.L. Proia was supported by the Intramural Research Program of the NIH, National Institute of Diabetes and Digestive and Kidney Diseases. T. Hla was supported by NIH grants HL67330, HL89934, CA77839, and HL117798. We also acknowledge the support of the Fondation LeDucq grant - SphingoNet.

Address correspondence to: Timothy Hla, Center for Vascular Biology, Department of Pathology and Laboratory Medicine, Weill Cornell Medical College, Cornell University, 1300 York Ave., New York, New York 10065, USA. Phone: 212.746.9953; E-mail: tih2002@med.cornell.edu. 
1. Hla T. Genomic insights into mediator lipidomics. Prostaglandins Other Lipid Mediat. 2005;77(1-4):197-209.

2. Spiegel S, Milstien S. Sphingosine-1-phosphate: an enigmatic signalling lipid. Nat Rev Mol Cell Biol. 2003;4(5):397-407.

3. Merrill AH Jr. Sphingolipid and glycosphingolipid metabolic pathways in the era of sphingolipidomics. Chem Rev. 2011;111(10):6387-6422.

4. Platt FM. Sphingolipid lysosomal storage disorders. Nature. 2014;510(7503):68-75.

5. Pitson SM, et al. Activation of sphingosine kinase 1 by ERK1/2-mediated phosphorylation. EMBO J. 2003;22(20):5491-5500.

6. Thompson CR, et al. Sphingosine kinase 1 (SK1) is recruited to nascent phagosomes in human macrophages: inhibition of SK1 translocation by Mycobacterium tuberculosis. JImmunol. 2005;174(6):3551-3561.

7. Shen $\mathrm{H}$, et al. Coupling between endocytosis and sphingosine kinase 1 recruitment. Nat Cell Biol. 2014;16(7):652-662.

8. Igarashi N, Okada T, Hayashi S, Fujita T, Jahangeer S, Nakamura S. Sphingosine kinase 2 is a nuclear protein and inhibits DNA synthesis. J Biol Chem. 2003;278(47):46832-46839.

9. Xiong Y, et al. Sphingosine kinases are not required for inflammatory responses in macrophages. J Biol Chem. 2013;288(45):32563-32573.

10. Mizugishi K, Yamashita T, Olivera A, Miller GF, Spiegel S, Proia RL. Essential role for sphingosine kinases in neural and vascular development. Mol Cell Biol. 2005;25(24):11113-11121.

11. Allende ML, et al. Mice deficient in sphingosine kinase 1 are rendered lymphopenic by FTY720. J Biol Chem. 2004;279(50):52487-52492.

12. Le Stunff H, Giussani P, Maceyka M, Lepine S, Milstien S, Spiegel S. Recycling of sphingosine is regulated by the concerted actions of sphingosine-1-phosphate phosphohydrolase 1 and sphingosine kinase 2.J Biol Chem. 2007;282(47):34372-34380.

13. Ogawa C, Kihara A, Gokoh M, Igarashi Y. Identification and characterization of a novel human sphingosine-1-phosphate phosphohydrolase, hSPP2. J Biol Chem. 2003;278(2):1268-1272.

14. Schulze H, Sandhoff K. Sphingolipids and lysosomal pathologies. Biochim Biophys Acta. 2014;1841(5):799-810.

15. Zhou J, Saba JD. Identification of the first mammalian sphingosine phosphate lyase gene and its functional expression in yeast. Biochem Biophys Res Commun. 1998;242(3):502-507.

16. Nakahara K, et al. The Sjogren-Larsson syndrome gene encodes a hexadecenal dehydrogenase of the sphingosine 1-phosphate degradation pathway. Mol Cell. 2012;46(4):461-471.

17. Bektas M, et al. Sphingosine 1-phosphate lyase deficiency disrupts lipid homeostasis in liver. J Biol Chem. 2010;285(14):10880-10889.

18. Dobrosotskaya IY, Seegmiller AC, Brown MS, Goldstein JL, Rawson RB. Regulation of SREBP processing and membrane lipid production by phospholipids in Drosophila. Science. 2002;296(5569):879-883.

19. Venkataraman K, et al. Vascular endothelium as a contributor of plasma sphingosine 1-phosphate. Circ Res. 2008;102(6):669-676.
20. Hisano Y, Kobayashi N, Yamaguchi A, Nishi T. Mouse SPNS2 functions as a sphingosine-1-phosphate transporter in vascular endothelial cells. PLoS One. 2012;7(6):e38941.

21. Pappu R, et al. Promotion of lymphocyte egress into blood and lymph by distinct sources of sphingosine-1-phosphate. Science. 2007;316(5822):295-298.

22. Yatomi Y, Ozaki Y, Ohmori T, Igarashi Y. Sphingosine 1-phosphate: synthesis and release. Prostaglandins. 2001;64(1-4):107-122.

23. Fukuhara $S$, et al. The sphingosine-1-phosphate transporter Spns2 expressed on endothelial cells regulates lymphocyte trafficking in mice. JClin Invest. 2012;122(4):1416-1426.

24. Jonnalagadda D, Sunkara M, Morris AJ, Whiteheart SW. Granule-mediated release of sphingosine-1-phosphate by activated platelets. Biochim Biophys Acta. 2014;1841(11):1581-1589.

25. Olivera A, Allende ML, Proia RL. Shaping the landscape: metabolic regulation of S1P gradients. Biochim Biophys Acta. 2013;1831(1):193-202.

26. Xiong Y, Yang P, Proia RL, Hla T. S1P as an essential erythrocrine factor for vascular development. JClin Invest. 2014;124(11):4823-4828.

27. Camerer E, et al. Sphingosine-1-phosphate in the plasma compartment regulates basal and inflammation-induced vascular leak in mice. JClin Invest. 2009;119(7):1871-1879.

28. Pham TH, et al. Lymphatic endothelial cell sphingosine kinase activity is required for lymphocyte egress and lymphatic patterning. J Exp Med. 2010;207(1):17-27.

29. Schwab SR, Pereira JP, Matloubian M, Xu Y, Huang Y, Cyster JG. Lymphocyte sequestration through S1P lyase inhibition and disruption of S1P gradients. Science. 2005;309(5741):1735-1739.

30. Allende ML, et al. Sphingosine-1-phosphate lyase deficiency produces a pro-inflammatory response while impairing neutrophil trafficking. J Biol Chem. 2011;286(9):7348-7358.

31. Breart B, et al. Lipid phosphate phosphatase 3 enables efficient thymic egress.J Exp Med. 2011;208(6):1267-1278.

32. Argraves KM, Argraves WS. HDL serves as a S1P signaling platform mediating a multitude of cardiovascular effects. J Lipid Res. 2007;48(11):2325-2333.

33. Christoffersen C, et al. Endothelium-protective sphingosine-1-phosphate provided by HDL-associated apolipoprotein M. Proc Natl Acad Sci U S A. 2011;108(23):9613-9618.

34. Blaho VA, Hla T. An update on the biology of sphingosine 1-phosphate receptors. J Lipid Res. 2014;55(8):1596-1608.

35. Regard JB, Sato IT, Coughlin SR. Anatomical profiling of $\mathrm{G}$ protein-coupled receptor expression. Cell. 2008;135(3):561-571.

36. Hla T, Maciag T. An abundant transcript induced in differentiating human endothelial cells encodes a polypeptide with structural similarities to G-protein-coupled receptors. J Biol Chem. 1990;265(16):9308-9313.

37. Chae SS, Proia RL, Hla T. Constitutive expression of the S1P1 receptor in adult tissues. Prostaglandins Other Lipid Mediat. 2004;73(1-2):141-150.

38. Chun J, Hla T, Lynch KR, Spiegel S, Moolenaar WH. International Union of Basic and Clinical Phar- macology. LXXVIII. Lysophospholipid receptor nomenclature. Pharmacol Rev. 2010;62(4):579-587.

39. Hanson MA, et al. Crystal structure of a lipid G protein-coupled receptor. Science. 2012;335(6070):851-855

40. Kono M, et al. The sphingosine-1-phosphate receptors S1P1, S1P2, and S1P3 function coordinately during embryonic angiogenesis. J Biol Chem. 2004;279(28):29367-29373.

41. Mendelson K, Zygmunt T, Torres-Vazquez J, Evans T, Hla T. Sphingosine 1-phosphate receptor signaling regulates proper embryonic vascular patterning. J Biol Chem. 2013;288(4):2143-2156

42. Kono M, Tucker AE, Tran J, Bergner JB, Turner EM, Proia RL. Sphingosine-1-phosphate receptor 1 reporter mice reveal receptor activation sites in vivo. JClin Invest. 2014;124(5):2076-2086.

43. Bischoff A, et al. Sphingosine-1-phosphate and sphingosylphosphorylcholine constrict renal and mesenteric microvessels in vitro. Br JPharmacol. 2000;130(8):1871-1877.

44. Murakami A, et al. Sphingosine 1-phosphate (S1P) regulates vascular contraction via S1P3 receptor: investigation based on a new S1P3 receptor antagonist. Mol Pharmacol. 2010;77(4):704-713.

45. Ohmori T, et al. Sphingosine 1-phosphate induces contraction of coronary artery smooth muscle cells via S1P2. Cardiovasc Res. 2003;58(1):170-177.

46. Tosaka M, et al. Sphingosine 1-phosphate contracts canine basilar arteries in vitro and in vivo: possible role in pathogenesis of cerebral vasospasm. Stroke. 2001;32(12):2913-2919.

47. Lorenz JN, Arend LJ, Robitz R, Paul RJ, MacLennan AJ. Vascular dysfunction in S1P2 sphingosine 1-phosphate receptor knockout mice. Am J Physiol Regul Integr Comp Physiol. 2007;292(1):R440-R446.

48. Kono M, et al. Deafness and stria vascularis defects in S1P2 receptor-null mice. J Biol Chem 2007;282(14):10690-10696

49. Herr DR, Grillet N, Schwander M, Rivera R, Muller U, Chun J. Sphingosine 1-phosphate (S1P) signaling is required for maintenance of hair cells mainly via activation of S1P2. J Neurosci. 2007;27(6):1474-1478.

50. MacLennan AJ, et al. The S1P2 sphingosine 1-phosphate receptor is essential for auditory and vestibular function. Hear Res. 2006;220(1-2):38-48.

51. Cyster JG, Schwab SR. Sphingosine-1-phosphate and lymphocyte egress from lymphoid organs. Annu Rev Immunol. 2012;30:69-94.

52. Juarez JG, et al. Sphingosine-1-phosphate facilitates trafficking of hematopoietic stem cells and their mobilization by CXCR 4 antagonists in mice. Blood. 2012;119(3):707-716.

53. Massberg S, et al. Immunosurveillance by hematopoietic progenitor cells trafficking through blood, lymph, and peripheral tissues. Cell. 2007;131(5):994-1008.

54. Jenne CN, et al. T-bet-dependent S1P5 expression in NK cells promotes egress from lymph nodes and bone marrow. J Exp Med. 2009;206(11):2469-2481.

55. Zhang L, et al. A novel role of sphingosine 1-phosphate receptor S1pr1 in mouse thrombopoiesis. JExp Med. 2012;209(12):2165-2181.

56. Lewis ND, et al. Circulating monocytes are reduced by sphingosine-1-phosphate receptor 
modulators independently of S1P3. JImmunol. 2013;190(7):3533-3540.

57. Thangada S, et al. Cell-surface residence of sphingosine 1-phosphate receptor 1 on lymphocytes determines lymphocyte egress kinetics. JExp Med. 2010;207(7):1475-1483.

58. Arnon TI, et al. GRK2-dependent S1PR1 desensitization is required for lymphocytes to overcome their attraction to blood. Science. 2011;333(6051):1898-1903.

59. Arnon TI, Horton RM, Grigorova IL, Cyster JG. Visualization of splenic marginal zone B-cell shuttling and follicular B-cell egress. Nature. 2013;493(7434):684-688

60. Green JA, et al. The sphingosine 1-phosphate receptor S1P(2) maintains the homeostasis of germinal center B cells and promotes niche confinement. Nat Immunol. 2011;12(7):672-680.

61. Cattoretti G, et al. Targeted disruption of the S1P2 sphingosine 1-phosphate receptor gene leads to diffuse large B-cell lymphoma formation. Cancer Res. 2009;69(22):8686-8692.

62. Lohr JG, et al. Discovery and prioritization of somatic mutations in diffuse large B-cell lymphoma (DLBCL) by whole-exome sequencing. Proc Natl Acad Sci U S A. 2012;109(10):3879-3884 .

63. Morin RD, et al. Mutational and structural analysis of diffuse large B-cell lymphoma using whole-genome sequencing. Blood. 2013;122(7):1256-1265.

64. Muppidi JR, et al. Loss of signalling via Galpha13 in germinal centre B-cell-derived lymphoma. Nature. 2014;516(7530):254-258.

65. Brinkmann V, et al. Fingolimod (FTY720): discovery and development of an oral drug to treat multiple sclerosis. Nat Rev Drug Discov. 2010;9(11):883-897.

66. Nijnik A, et al. The role of sphingosine-1-phosphate transporter Spns 2 in immune system function. JImmunol. 2012;189(1):102-111.

67. Mendoza A, et al. The transporter Spns2 is required for secretion of lymph but not plasma sphingosine-1-phosphate. Cell Rep. 2012;2(5):1104-1110.

68. Nagahashi M, et al. Spns2, a transporter of phosphorylated sphingoid bases, regulates their blood and lymph levels, and the lymphatic network. FASEB J. 2013;27(3):1001-1011.

69. Vogel P, et al. Incomplete inhibition of sphingosine 1-phosphate lyase modulates immune system function yet prevents early lethality and nonlymphoid lesions. PLoS One. 2009;4(1):e4112.

70. Billich A, et al. Partial deficiency of sphingosine-1-phosphate lyase confers protection in experimental autoimmune encephalomyelitis. PLoS One. 2013;8(3):e59630.

71. Chun J, Hartung HP. Mechanism of action of oral fingolimod (FTY720) in multiple sclerosis. Clin Neuropharmacol. 2010;33(2):91-101.

72. Chan JP, Hu Z, Sieburth D. Recruitment of sphingosine kinase to presynaptic terminals by a conserved muscarinic signaling pathway promotes neurotransmitter release. Genes Dev. 2012;26(10):1070-1085.

73. Kajimoto T, Okada T, Yu H, Goparaju SK, Jahangeer S, Nakamura S. Involvement of sphingosine-1-phosphate in glutamate secretion in hippocampal neurons. Mol Cell Biol.
2007;27(9):3429-3440

74. Kanno T, et al. Regulation of synaptic strength by sphingosine 1-phosphate in the hippocampus. Neuroscience. 2010;171(4):973-980.

75. Choi JW, et al. FTY720 (fingolimod) efficacy in an animal model of multiple sclerosis requires astrocyte sphingosine 1-phosphate receptor 1 (S1P1) modulation. Proc Natl Acad Sci U S A. 2011;108(2):751-756.

76. Wu YP, Mizugishi K, Bektas M, Sandhoff R, Proia RL. Sphingosine kinase 1/S1P receptor signaling axis controls glial proliferation in mice with Sandhoff disease. Hum Mol Genet. 2008;17(15):2257-2264.

77. Coste O, et al. Sphingosine 1-phosphate modulates spinal nociceptive processing. J Biol Chem. 2008;283(47):32442-32451.

78. Camprubi-Robles M, et al. Sphingosine-1phosphate-induced nociceptor excitation and ongoing pain behavior in mice and humans is largely mediated by S1P3 receptor. J Neurosci. 2013;33(6):2582-2592.

79. Welch SP, Sim-Selley LJ, Selley DE. Sphingosine-1-phosphate receptors as emerging targets for treatment of pain. Biochem Pharmacol. 2012;84(12):1551-1562.

80. Herzog BH, et al. Podoplanin maintains high endothelial venule integrity by interacting with platelet CLEC-2. Nature. 2013;502(7469):105-109.

81. Ikeda $\mathrm{H}$, et al. Sphingosine 1-phosphate regulates regeneration and fibrosis after liver injury via sphingosine 1-phosphate receptor 2. J Lipid Res. 2009;50(3):556-564.

82. Shea BS, Brooks SF, Fontaine BA, Chun J, Luster AD, Tager AM. Prolonged exposure to sphingosine 1-phosphate receptor-1 agonists exacerbates vascular leak, fibrosis, and mortality after lung injury. Am J Respir Cell Mol Biol. 2010;43(6):662-673.

83. Schwalm S, Pfeilschifter J, Huwiler A. Sphingosine-1-phosphate: a Janus-faced mediator of fibrotic diseases. Biochim Biophys Acta. 2013;1831(1):239-250

84. Pelletier D, Hafler DA. Fingolimod for multiple sclerosis. N EnglJ Med.2012;366(4):339-347.

85. Garris CS, et al. Defective sphingosine 1-phosphate receptor 1 (S1P1) phosphorylation exacerbates TH17-mediated autoimmune neuroinflammation. Nat Immunol. 2013;14(11):1166-1172.

86. Cruz-Orengo L, et al. Enhanced sphingosine-1phosphate receptor 2 expression underlies female CNS autoimmunity susceptibility. JClin Invest. 2014;124(6):2571-2584.

87. Sanchez T, Skoura A, Wu MT, Casserly B, Harrington EO, Hla T. Induction of vascular permeability by the sphingosine-1-phosphate receptor-2 (S1P2R) and its downstream effectors ROCK and PTEN. Arterioscler Thromb Vasc Biol. 2007;27(6):1312-1318.

88. Michaud J, Im DS, Hla T. Inhibitory role of sphingosine 1-phosphate receptor 2 in macrophage recruitment during inflammation. JImmunol. 2010;184(3):1475-1483.

89. Holleran WM, Takagi Y, Uchida Y. Epidermal sphingolipids: metabolism, function, and roles in skin disorders. FEBS Lett. 2006;580(23):5456-5466.

90. Yamashita T, et al. Interruption of ganglioside synthesis produces central nervous system degeneration and altered axon-glial interactions. Proc Natl Acad Sci U S A. 2005;102(8):2725-2730.

91. Teijaro JR, et al. Endothelial cells are central orchestrators of cytokine amplification during influenza virus infection. Cell. 2011;146(6):980-991.

92. Teijaro JR, et al. Protection of ferrets from pulmonary injury due to H1N1 2009 influenza virus infection: immunopathology tractable by sphingosine-1-phosphate 1 receptor agonist therapy. Virology. 2014;452-453:152-157.

93. Teijaro JR, Walsh KB, Rice S, Rosen H, Oldstone MB. Mapping the innate signaling cascade essential for cytokine storm during influenza virus infection. Proc Natl Acad Sci U S A. 2014;111(10):3799-3804

94. Matthay MA, Ware LB, Zimmerman GA. The acute respiratory distress syndrome. JClin Invest. 2012;122(8):2731-2740.

95. McVerry BJ, Peng X, Hassoun PM, Sammani S, Simon BA, Garcia JG. Sphingosine 1-phosphate reduces vascular leak in murine and canine models of acute lung injury. Am J Respir Crit Care Med. 2004;170(9):987-993.

96. Peng $X$, et al. Protective effects of sphingosine 1-phosphate in murine endotoxin-induced inflammatory lung injury. Am J Respir Crit Care Med. 2004;169(11):1245-1251.

97. Oo ML, et al. Engagement of S1P(1)-degradative mechanisms leads to vascular leak in mice. J Clin Invest. 2011;121(6):2290-2300.

98. Zhang Y, et al. Elevated sphingosine-1-phosphate promotes sickling and sickle cell disease progression. J Clin Invest. 2014;124(6):2750-2761.

99. Platt OS. Sickle cell anemia as an inflammatory disease. J Clin Invest. 2000;106(3):337-338.

100.Chae SS, Paik JH, Furneaux H, Hla T. Requirement for sphingosine 1-phosphate receptor- 1 in tumor angiogenesis demonstrated by in vivo RNA interference. J Clin Invest. 2004;114(8):1082-1089.

101.Lee $\mathrm{H}$, et al. STAT3-induced S1PR1 expression is crucial for persistent STAT3 activation in tumors. Nat Med.2010;16(12):1421-1428.

102. Liang J, et al. Sphingosine-1-phosphate links persistent STAT3 activation, chronic intestinal inflammation, and development of colitis-associated cancer. Cancer Cell. 2013;23(1):107-120.

103. Ponnusamy S, et al. Communication between host organism and cancer cells is transduced by systemic sphingosine kinase $1 /$ sphingosine 1-phosphate signalling to regulate tumour metastasis. EMBO Mol Med. 2012;4(8):761-775.

104. Hanahan D, Weinberg RA. Hallmarks of cancer: the next generation. Cell. 2011;144(5):646-674.

105. Pyne NJ, Pyne S. Sphingosine 1-phosphate and cancer. Nat Rev Cancer. 2010;10(7):489-503.

106.Visentin B, et al. Validation of an anti-sphingosine-1-phosphate antibody as a potential therapeutic in reducing growth, invasion, and angiogenesis in multiple tumor lineages. Cancer Cell. 2006;9(3):225-238.

107. Xia P, et al. An oncogenic role of sphingosine kinase. Curr Biol. 2000;10(23):1527-1530.

108. Kharel Y, et al. Sphingosine kinase type 1 inhibition reveals rapid turnover of circulating sphingosine 1-phosphate. Biochem J. 2011;440(3):345-353. 
109. Rex K, et al. Sphingosine kinase activity is not required for tumor cell viability. PLoS One. 2013;8(7):e68328.

110.Schnute ME, et al. Modulation of cellular S1P levels with a novel, potent and specific inhibitor of sphingosine kinase-1. Biochem J. 2012;444(1):79-88.

111. Snider AJ, et al. A role for sphingosine kinase 1 in dextran sulfate sodium-induced colitis. FASEB J. 2009;23(1):143-152.

112. Daniel C, Sartory N, Zahn N, Geisslinger G, Radeke HH, Stein JM. FTY720 ameliorates Th1mediated colitis in mice by directly affecting the functional activity of $\mathrm{CD} 4{ }^{+} \mathrm{CD} 25^{+}$regulatory $\mathrm{T}$ cells. J Immunol. 2007;178(4):2458-2468.

113. Daniel C, et al. FTY720 ameliorates oxazolone colitis in mice by directly affecting Thelper type 2 functions. Mol Immunol.
2007;44(13):3305-3316.

114. Montrose DC, et al. S1P(1) localizes to the colonic vasculature in ulcerative colitis and maintains blood vessel integrity. J Lipid Res. 2013;54(3):843-851.

115. Keul P, et al. The sphingosine-1-phosphate analogue FTY720 reduces atherosclerosis in apolipoprotein E-deficient mice. Arterioscler Thromb Vasc Biol. 2007;27(3):607-613.

116. Poti F, et al. KRP-203, sphingosine 1-phosphate receptor type 1 agonist, ameliorates atherosclerosis in LDL-R $\mathrm{R}^{-/}$mice. Arterioscler Thromb Vasc Biol. 2013;33(7):1505-1512.

117. Skoura A, et al. Sphingosine-1-phosphate receptor-2 function in myeloid cells regulates vascular inflammation and atherosclerosis. Arterioscler Thromb Vasc Biol. 2011;31(1):81-85.

118. Keul P, et al. Sphingosine-1-phosphate receptor
3 promotes recruitment of monocyte/macrophages in inflammation and atherosclerosis. Circ Res. 2011;108(3):314-323.

119. Jin ZQ, Goetzl EJ, Karliner JS. Sphingosine kinase activation mediates ischemic preconditioning in murine heart. Circulation. 2004;110(14):1980-1989.

120. Jin ZQ, Zhang J, Huang Y, Hoover HE, Vessey DA, Karliner JS. A sphingosine kinase 1 mutation sensitizes the myocardium to ischemia/reperfusion injury. Cardiovasc Res. 2007;76(1):41-50.

121. Ochi R, Momose Y, Oyama K, Giles WR. Sphingosine-1-phosphate effects on guinea pig atrial myocytes: Alterations in action potentials and $\mathrm{K}+$ currents. Cardiovasc Res. 2006;70(1):88-96.

122. Means CK, Brown JH. Sphingosine-1-phosphate receptor signalling in the heart. Cardiovasc Res. 2009;82(2):193-200. 